\title{
Influence of Magnetohydrodynamic and Thermal Radiation Boundary Layer Flow of a Nanofluid Past a Stretching Sheet
}

\author{
M. G. Reddy* \\ Department of Mathematics, Acharya Nagarjuna University Campus, Ongole, A.P. (India)-523 001
}

Received 1 December 2013, accepted in final revised form 23 January 2014

\begin{abstract}
The problem of laminar fluid flow which results from a permeable stretching of a flat surface in a nanofluid with the effects of heat radiation, magnetic field, velocity slip and convective boundary conditions have been investigated. The transport equations used in the analysis took into account the effect of Brownian motion and thermophoresis parameters. The solution for the velocity, temperature and nanoparticle concentration depends on parameters viz. thermal radiation parameter $R$, magnetic parameter $M$, Prandtl number Pr, Lewis number $L e$, Brownian motion parameter $N b$, thermophoresis parameter $N t$, velocity slip parameter $A$ convection and Biot numbe $B i$. Similarity transformation is used to convert the governing non-linear boundary-layer equations into coupled higher order nonlinear ordinary differential equations. These equations are numerically solved using fourth order Runge-Kutta method along with shooting technique. An analysis has been carried out to elucidate the effects of governing parameters corresponding to various physical conditions. Numerical results are obtained for distributions of velocity, temperature and concentration, as well as, for the skin friction, local Nusselt number and local Sherwood number for several values of governing parameters.
\end{abstract}

Keywords: Nanofluid, Boundary layer flow; Stretching sheet; Thermal radiation; MHD; Velocity slip; Convective boundary.

(C) 2014 JSR Publications. ISSN: 2070-0237 (Print); 2070-0245 (Online). All rights reserved.

doi: http://dx.doi.org/10.3329/jsr.v6i2.17233 $\quad$ J. Sci. Res. 6 (2), 257-272 (2014)

\section{Introduction}

The flow over a stretching surface is an important problem in many engineering processes with applications in industries such as extrusion, melt-spinning, the hot rolling, wire drawing, glass- fiber production, manufacture of plastic and rubber sheets, cooling of a large metallic plate in a bath, which may be an electrolyte, etc. In industry, polymer sheets and filaments are manufactured by continuous extrusion of the polymer from a die to a windup roller, which is located at a finite distance away. The thin polymer sheet constitutes a continuously moving surface with a non-uniform velocity through an

\footnotetext{
*Email: mgrmaths@gmail.com
} 
ambient fluid [1]. Experiments show that the velocity of the stretching surface is approximately proportional to the distance from the orifice [2]. Crane [3] studied the steady two-dimensional incompressible boundary layer flow of a Newtonian fluid caused by the stretching of an elastic flat sheet which moves in its own plane with a velocity varying linearly with the distance from a fixed point due to the application of a uniform stress. This problem is particularly interesting since an exact solution of the twodimensional Navier-Stokes equations has been obtained by Crane [3]. After this pioneering work, the flow field over a stretching surface has drawn considerable attention and a good amount of literature has been generated on this problem [4-9].

Nanofluids are the suspension of nanometer-sized solid particles and fibers, which have been proposed as a means for enhancing the performance of heat transfer liquids currently available, such as water, toluene, oil and ethylene glycol mixture. Nanofluids have received the interest of many researchers recently because of their greatly enhanced thermal conductivity property [10]. One can refer the works of authors [11-13] regarding the thermal conductivity enhancement of the nanofluids available in the literature. Nowadays, the study of convective heat transfer in nanofluids become active research area due to its heat transfer enhancement characteristics. Because of the fact that cooling is one of the technical challenges faced many industries including microelectronic, transportation, solid-state lighting and manufacturing; the idea of nanofluid has been proposed as a means of alleviating these challenges.

The boundary layer flow and heat transfer due to nanofluids over a stretching sheet are a thrust areas of current research. Such investigations find applications over a broad spectrum of science and engineering disciplines. An important aspect of boundary layer flow of a nanofluid over a stretching sheet is the heat transfer characteristics. It is crucial to understand the heat transfer characteristics of the stretching sheet so that the finished product meets the desired quality. This is due to the fact that the quality of a final product depends on the rate of heat transfer at the stretching surface. Accordingly, Kuznetsov and Nield [14] have studied the natural convective boundary-layer flow of a nanofluid past a vertical plate analytically. They used a model in which Brownian motion and thermophoresis effects were taken into account. Moreover, Khan and Pop [15] used the same model to study the boundary layer flow of a nanofluid past a stretching sheet with a constant surface temperature. Very recently Ibrahim and Shanker [16] have studied the boundary-layer flow and heat transfer of nanofluid over a vertical Plate taking into account the convective surface boundary condition. Recently, Haddad et al. [17] experimentally investigated natural convection in nanofluid by considering the role of thermophoresis and Brownian motion in heat transfer enhancement. They indicated that neglecting the role of Brownian motion and thermophoresis deteriorate the heat transfer and this deterioration elevates when the volume fraction of a nanoparticles increases. Makinde and Aziz [18] to study boundary layer flow of a nanofluid past a stretching sheet with a convective boundary condition.

In all the previous investigations, the effects of radiation on the flow and heat transfer have not been studied. It is well known that radiative heat transfer flow is very important 
in manufacturing industries for the design of reliable equipments, nuclear plants, gas turbines and various propulsion devices for aircraft, missiles, satellites and space vehicles. Also, the effects of thermal radiation on forced and free convection flow are important in the content of space technology and process involving high temperature. Gnaneswara Reddy [19] analyzed heat generation and thermal radiation effects over a stretching sheet in a micropolar fluid. Olanrewaju et al. [20] studied the boundary layer flow of nanofluids over a moving surface in a flowing fluid in the presence of radiation. Thermophoresis effects on MHD combined heat and mass transfer in two-dimensional flow over an inclined radiative isothermal permeable surface was investigated by $\mathrm{M}$. Gnaneswara Reddy [21]. Akbar et al. [22] considered the radiation effects on MHD stagnation point flow of nano fluid towards a stretching surface with convective boundary condition. Very Recently, Nadeem et al. [23] studied the heat transfer analysis of water-based nanofluid over an exponentially stretching sheet

The purpose of present paper is to examine the influence of magnetohydrodynamic (MHD) and heat radiation on boundary layer flow, heat transfer and nanoparticle fraction over a stretching surface in a nanofluid with velocity slip and convective boundary conditions. The governing boundary layer equations have been transformed to a two-point boundary value problem in similarity variables, and these have been solved numerically. The effects of embedded parameters on fluid velocity, temperature and particle concentration have been shown graphically.

\section{Convective Transport Equations}

Consider steady two-dimensional steady boundary layer flow of a nanofluid past a stretching sheet with a linear velocity variation with the distance $x$ i.e. $u_{w}=a x$ where $a$ is a real positive number and $x$ is the coordinate measured from the location where the sheet velocity is zero.

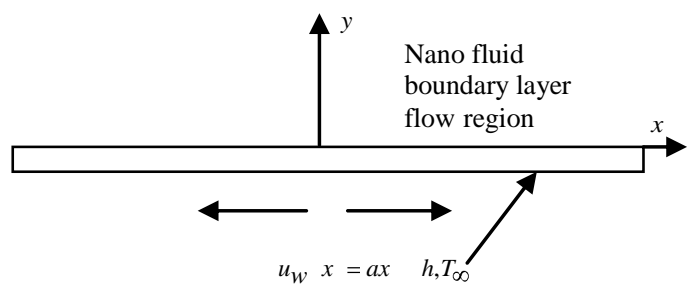

Fig. 1. Physical model and coordinate system.

The sheet surface temperature $T_{w}$, to be determined later, is the result of a convective heating process which is characterized by a temperature $T_{f}$ and a heat transfer coefficient $h$. The nanoparticle volume fraction $C$ at the wall is $C_{w}$, while at large values of $y$, the value is $C_{\infty}$. The flow is assumed to be generated by stretching sheet issuing from a thin 
slit at the origin. The sheet is then stretched in such a way that the speed at any point on the sheet becomes proportional to the distance from the origin. The ambient temperature and concentration, respectively, are $T_{\infty}$ and $C_{\infty}$. The flow is subjected to the effect of thermal radiation and a transverse magnetic field of strength $B_{0}$, which is assumed to be applied in the positive $y$ direction, normal to the surface. The induced magnetic field is also assumed to be small compared to the applied magnetic field; so it is neglected. It is further assumed that the base fluid and the suspended nanoparticles are in thermal equilibrium. It is chosen that the coordinate system $x$-axis is along stretching sheet and $y$-axis is normal to the sheet.

Under the above assumptions, the governing equation of the conservation of mass, momentum, energy and nanoparticles fraction in the presence of magnetic field and thermal radiation past a stretching sheet can be expressed as follows [15 ]:

$$
\begin{aligned}
& \frac{\partial u}{\partial x}+\frac{\partial v}{\partial y}=0 \\
& u \frac{\partial u}{\partial x}+v \frac{\partial u}{\partial y}=-\frac{1}{\rho_{f}} \frac{\partial p}{\partial x}+v\left(\frac{\partial^{2} u}{\partial x^{2}}+\frac{\partial^{2} u}{\partial y^{2}}\right)-\frac{\sigma B_{0}^{2}}{\rho_{f}} u \\
& u \frac{\partial v}{\partial x}+v \frac{\partial v}{\partial y}=-\frac{1}{\rho_{f}} \frac{\partial p}{\partial y}+v\left(\frac{\partial^{2} v}{\partial x^{2}}+\frac{\partial^{2} v}{\partial y^{2}}\right)-\frac{\sigma B_{0}^{2}}{\rho_{f}} v \\
& \frac{\partial T}{\partial x}+v \frac{\partial T}{\partial y}=\alpha \nabla^{2} T-\frac{1}{\rho c_{f}} \frac{\partial q_{r}}{\partial y} \\
& +\tau\left\{D_{B}\left(\frac{\partial C}{\partial x} \frac{\partial T}{\partial x}+\frac{\partial C}{\partial y} \frac{\partial T}{\partial y}\right)+\left(\frac{D_{T}}{T_{\infty}}\right)\left[\left(\frac{\partial T}{\partial x}\right)^{2}+\left(\frac{\partial T}{\partial y}\right)^{2}\right]\right\} \\
& u \frac{\partial C}{\partial x}+v \frac{\partial C}{\partial y}=D_{B}\left(\frac{\partial^{2} C}{\partial x^{2}}+\frac{\partial^{2} C}{\partial y^{2}}\right)+\left(\frac{D_{T}}{T_{\infty}}\right)\left(\frac{\partial^{2} T}{\partial x^{2}}+\frac{\partial^{2} T}{\partial y^{2}}\right)
\end{aligned}
$$

where $u$ and $v$ are the velocity components along the $x, y$ directions, respectively, $\rho_{f}$ is the density of base fluid, $\sigma$ is the electrical conductivity, $B_{0}$ is the magnetic field $\mu$ is the absolute viscosity of the base fluid, $g$ is the acceleration due to gravity, $\alpha=\frac{k}{\rho c_{f}}$ is the thermal diffusivity of the base fluid, $\tau=\frac{\rho c_{p}}{\rho c_{f}}$ is the ratio of nanoparticle heat capacity and the base fluid heat capacity, $C$ is the local nanoparticle volume fraction, $\beta$ is volumetric thermal expansion coefficient of the base fluid, $D_{B}$ is the Brownian diffusion coefficient, $D_{T}$ is the thermophoretic diffusion coefficient, $T$ is the local temperature, $\rho$ is the density, $T_{\infty}$ is the temperature far away from the sheet, and $q_{r}$ is the radiative heat flux 
in the $y$-direction. The subscript $\infty$ denotes the values at large values of $y$ where the fluid is quiescent.

The appropriate boundary conditions for the physical problem under consideration are given by

$$
\begin{aligned}
& u=u_{w}+L \frac{\partial u}{\partial y}, v=v_{w},-k \frac{\partial T}{\partial y}=h T_{f}-T, C=C_{w} \text { at } y=0 \\
& u \rightarrow 0, T \rightarrow T_{\infty}, C \rightarrow C_{\infty} \text { as } y \rightarrow \infty
\end{aligned}
$$

$u_{w}=a x, L$ is the velocity slip factor, $v_{w}>0$ corresponds to suction velocity whereas $v_{w}<0$ shows the injection or blowing,

Using an order magnitude analysis of the $y$-direction momentum equation (normal to the sheet) and the usual boundary layer approximations, such as:

$$
\begin{aligned}
& u \square v \\
& \frac{\partial u}{\partial y} \square \frac{\partial u}{\partial x}, \frac{\partial v}{\partial x}, \frac{\partial v}{\partial y} \\
& \frac{\partial p}{\partial y}=0
\end{aligned}
$$

After boundary-layer approximation, the governing equations are reduced to:

$$
\begin{aligned}
& \frac{\partial u}{\partial x}+\frac{\partial v}{\partial y}=0 \\
& u \frac{\partial u}{\partial x}+v \frac{\partial u}{\partial y}=-\frac{1}{\rho_{f}} \frac{\partial p}{\partial x}+v\left(\frac{\partial^{2} u}{\partial x^{2}}+\frac{\partial^{2} u}{\partial y^{2}}\right)-\frac{\sigma B_{0}^{2}}{\rho_{f}} u \\
& \frac{\partial T}{\partial x}+v \frac{\partial T}{\partial y}=\alpha \nabla^{2} T-\frac{1}{\rho c_{f}} \frac{\partial q_{r}}{\partial y} \\
& +\tau\left\{D_{B}\left(\frac{\partial C}{\partial x} \frac{\partial T}{\partial x}+\frac{\partial C}{\partial y} \frac{\partial T}{\partial y}\right)+\left(\frac{D_{T}}{T_{\infty}}\right)\left[\left(\frac{\partial T}{\partial x}\right)^{2}+\left(\frac{\partial T}{\partial y}\right)^{2}\right]\right\} \\
& u \frac{\partial C}{\partial x}+v \frac{\partial C}{\partial y}=D_{B}\left(\frac{\partial^{2} C}{\partial x^{2}}+\frac{\partial^{2} C}{\partial y^{2}}\right)+\left(\frac{D_{T}}{T_{\infty}}\right)\left(\frac{\partial^{2} T}{\partial x^{2}}+\frac{\partial^{2} T}{\partial y^{2}}\right)
\end{aligned}
$$

The boundary conditions are:

$$
\begin{aligned}
& u=u_{w}+L \frac{\partial u}{\partial y}, v=v_{w},-k \frac{\partial T}{\partial y}=h T_{f}-T, C=C_{w} \text { at } y=0 \\
& u \rightarrow 0, T \rightarrow T_{\infty}, C \rightarrow C_{\infty} \text { as } y \rightarrow \infty
\end{aligned}
$$

Introducing the following dimensionless quantities, the mathematical analysis of the problem is simplified by using similarity transforms: 


$$
\eta=a / v^{1 / 2} y, \psi=a v^{1 / 2} x f \eta, \theta \eta=\frac{T-T_{\infty}}{T_{f}-T_{\infty}}, \phi \eta=\frac{C-C_{\infty}}{C_{w}-C_{\infty}}
$$

The equation of continuity is satisfied if a stream function $\psi x, y$ is chosen as:

$$
u=\frac{\partial \psi}{\partial y}, v=-\frac{\partial \psi}{\partial x}
$$

The radiative heat flux in the $x$-direction is considered negligible as compared to $y$ direction. Hence, by using Rosseland approximation for radiation, the radiative heat flux $q_{r}$ is given by

$q_{r}=-\frac{4 \sigma_{s}}{3 k_{e}} \frac{\partial T^{4}}{\partial y}$

where $\sigma_{s}$ is the Stefan-Boltzmann constant and $k_{e}$ - the mean absorption coefficient. It should be noted that by using the Rosseland approximation, the present analysis is limited to optically thick fluids. If the temperature differences within the flow are sufficiently small, then Eq. (15) can be linearized by expanding $T^{4}$ into the Taylor series about $T_{\infty}$, which after neglecting higher order terms takes the form

$$
T^{4} \cong 4 T_{\infty}^{3} T-3 T_{\infty}^{4}
$$

Thus, substituting Eq. (16) into Eq. (15), we get:

$$
q_{r}=-\frac{16 T_{\infty}^{3} \sigma_{s}}{3 k_{e}} \frac{\partial^{2} T^{4}}{\partial y^{2}}
$$

Using the similarity transformation quantities, the governing Eqs. (8)-(11) are transformed to the ordinary differential equation as follows:

$$
\begin{aligned}
& f^{\prime \prime \prime}+f f^{\prime \prime}-f^{\prime 2}-M f^{\prime}=0 \\
& \left(1+\frac{4}{3} R\right) \theta^{\prime \prime}+\operatorname{Pr} f \theta^{\prime}+\operatorname{Pr} N t \theta^{2}+\operatorname{Pr} N b \phi^{\prime} \theta^{\prime}=0 \\
& \phi^{\prime \prime}+\operatorname{Pr} \operatorname{Lef} \phi^{\prime}+\frac{N t}{N b} \theta^{\prime \prime}=0
\end{aligned}
$$

The corresponding boundary conditions take the form

$$
\begin{aligned}
& f 0=S, f^{\prime} 0=1+A f^{\prime \prime} 0, \theta^{\prime} 0=-B i 1-\theta 0 \quad, \phi 0=1 \\
& f^{\prime} \infty=0, \theta \infty=0, \phi \infty=0
\end{aligned}
$$

where the governing parameters are defined as:

$$
\operatorname{Pr}=\frac{v}{\alpha}, \quad N b=\frac{\rho c_{p} D_{B} C_{w}-C_{\infty}}{\rho c_{f} v}, N t=\frac{\rho c_{p} D_{B} T_{f}-T_{\infty}}{\rho c_{f} v T_{\infty}}, B i=\frac{h v / a^{1 / 2}}{k},
$$




$$
M=\frac{\sigma B_{0}^{2}}{\rho_{f} a}, \quad R=\frac{4 \sigma_{s} T_{\infty}^{3}}{3 k k_{e}}, L e=\frac{\alpha}{D_{B}}, \quad A=L a / v^{1 / 2}, S=-\frac{v_{w}}{a v^{1 / 2}}
$$

where $f^{\prime}, \theta$ and $\phi$ are the dimensionless velocity, temperature and nanoparticle concentration, respectively. $\eta$ is the similarity variables, the prime denotes differentiation with respect to $\eta$. $P r, R, M, N b, N t, A$ and $S$ denote a Prandtl number, a radiation parameter, a magnetic parameter, a Brownian motion parameter, a thermophoresis parameter, a Lewis number, a the velocity slip parameter and a suction parameter respectively.

The important physical quantities of interest in this problem are local skin friction coefficient $C_{f}$, the local Nusselt number $N u_{x}$ and the local Sherwood number $S h_{x}$ are defined as:

$$
C_{f}=\frac{\tau_{w}}{\rho u_{w}^{2}}, \quad N u_{x}=\frac{x q_{w}}{k T_{w}-T_{\infty}}, \quad S h_{x}=\frac{x q_{m}}{D_{B} \phi_{w}-\phi_{\infty}}
$$

Where the wall heat flux $q_{w}$ and mass flux $q_{m}$ are given by:

$$
q_{w}=-k\left(\frac{\partial T}{\partial y}\right)_{y=0}, q_{m}=-D_{B}\left(\frac{\partial C}{\partial y}\right)_{y=0}
$$

By using the above equations, we get:

$$
C_{f} \operatorname{Re}_{x}^{1 / 2}=-f^{\prime \prime} 0, \frac{N u_{x}}{\operatorname{Re}_{x}{ }^{1 / 2}}=-1+R \quad \theta^{\prime} 0, \frac{S h_{x}}{\operatorname{Re}_{x}{ }^{1 / 2}}=-\phi^{\prime} 0
$$

where $C_{f}, R e_{x}, N u_{x}, S h_{x}$ are the skin friction, local Reynolds number, local Nusselt number and local Sherwood number, respectively.

\section{Method of Solution}

An efficient fourth order Runge-Kutta method along with shooting technique has been employed to study the flow model for the above coupled non-linear ordinary differential Eqs. (18)-(20) for different values of governing parameters viz. magnetic parameter $M$, Prandtl number Pr, a radiation parameter $R$, a Brownian motion parameter $N b$, a thermophoresis parameter $N t$ and a Lewis number Le. The non-linear differential equations are first decomposed into a system of first order differential equation. The coupled ordinary differential Eqs. (18)-(20) are third order in $f$ and second order in $\theta$ and $\phi$ which have been reduced to a system of seven simultaneous equations for six unknowns. In order to numerically solve this system of equations using Runge-Kutta method, the solution requires seven initial conditions but two initial conditions in $f$ one initial condition in each of $\theta$ and $\phi$ are known. However, the values of $f^{\prime}, \theta$ and $\phi$ are known at $\eta \rightarrow \infty$. These end conditions are utilized to produce unknown initial conditions at $\eta=0$ by using shooting technique. The most important step of this scheme is to choose the appropriate finite value of $\eta_{\infty}$. Thus to estimate the value of $\eta_{\infty}$, we start with some initial guess value and solve the boundary value problem consisting of Eqs. (18)-(20) to obtain $f^{\prime \prime} 0, \theta^{\prime} \quad 0$ and $\phi^{\prime} 0$. The solution process is repeated with another larger value 
of $\eta_{\infty}$ until two successive values of $f^{\prime \prime} 0, \theta^{\prime} 0$ and $\phi^{\prime} 0$ differ only after desired significant digit. The last value $\eta_{\infty}$ is taken as the finite value of the limit $\eta_{\infty}$ for the particular set of physical parameters for determining velocity, temperature and concentration, respectively, are $f \eta, \theta \eta$ and $\phi \eta$ in the boundary layer. After getting all the initial conditions we solve this system of simultaneous equations using fourth order Runge-Kutta integration scheme. The value of $\eta_{\infty}$ is selected to vary from 5 to 6 depending on the physical parameters governing the flow so that no numerical oscillation would occur.

\section{Results and Discussion}

The aim of this section is to analyze the effects of various physical parameters on the velocity, temperature and nanoparticle concentration fields, respectively. The numerical solutions are obtained for velocity, temperature and concentration profiles for different values of governing parameters. The obtained results are displayed through graphs Figs. 2-16 for velocity, temperature and concentration profiles, respectively.

In the present study following default parameter values are adopted for computations: $\operatorname{Pr}=2.0, M=2.0, R=2.0, N b=N t=S=A=0.5, B i=0.1$ and $L e=5.0$. All graphs therefore correspond to these values unless specifically indicated on the appropriate graph and table.

First, a comparison with previously published papers available in the literature has been done in order to check the accuracy of the present results for different values of Pr in Table 1. It is notice that the comparison shows good agreement for each value of $\operatorname{Pr}$. Therefore, we are confident that the present results are very accurate.

Table 1. Comparison of results for the reduced Nusselt number $-\theta(0)$ in the absence of nanofluid with $M=R=0$ and $B i=1000$.

\begin{tabular}{ccccc}
\hline $\operatorname{Pr}$ & $\begin{array}{c}\text { Khan and Pop } \\
{[15]}\end{array}$ & Wang [22] & $\begin{array}{c}\text { Makinde and } \\
\text { Aziz [18] }\end{array}$ & $\begin{array}{c}\text { Present } \\
\text { result }\end{array}$ \\
\hline 0.07 & 0.0663 & 0.0656 & 0.0656 & 0.0656 \\
0.20 & 0.1691 & 0.1691 & 0.1691 & 0.1691 \\
0.70 & 0.4539 & 0.4539 & 0.4539 & 0.4539 \\
2.00 & 0.9113 & 0.9114 & 0.9114 & 0.9113 \\
7.00 & 1.8954 & 1.8954 & 1.8954 & 1.8954 \\
\hline
\end{tabular}

Table 2 presents the variation of the skin friction coefficient in relation to magnetic field, suction and velocity slip parameters. On observing this table, as both the values of magnetic field and suction parameters increase, the values of skin friction coefficient increase. However, the skin friction coefficient decreases as the values of velocity slip parameter increase. Table 3 has been prepared to illustrate the effects Brownian motion 
parameter, a thermophoresis parameter on the local Nusselt and Sherwood numbers. The first two entries show that for a fixed thermophoresis parameter, $N t=0.1$, local Nusselt number decreases sharply with the increase in Brownian motion, that as $N b$ is increased from 0.1 to 0.5 . However, the reduced Sherwood number increases substantially as $N b$ is increased from 0.1 to 0.5 but tends to plateau beyond $N b=0.5$. These observations are consistent with the initial slopes of the temperature and concentration profiles to be discussed later. As the Brownian motion intensifies, it impacts a larger extent of the fluid, causing the thermal boundary layer to thicken, which in turn decreases the local Nusselt number. The thickening of the boundary layer due to stronger Brownian motion will be highlighted again when the temperature profiles are discussed. The last two entries in Table 3 show that the local Nusselt number decreases as the thermophoresis diffusion penetrates deeper into the fluid and causes the thermal boundary layer to thicken. However, the increase in the thermophoresis parameter enhances the Sherwood number. Table 4 has been prepared to illustrate the effects of Prandtl number, radiation parameter, Biot number and Lewis number, on the local Nusselt and Sherwood numbers of the stretching sheet. On observing this table, as the values of $R$ parameter increase, the values of $-\theta^{\prime} 0$ decrease but $-\phi^{\prime} 0$ increase. It is observed that as the Prandtl number increases further, there is a significant decrease in local Nusselt number and a significant increase in local Sherwood number. Also, an increase in Lewis number causes the reduced Nusselt number to decrease but increase the reduced Sherwood number.

Table 2. Computed values of skin friction coefficient $-f^{\prime \prime} 0$ for various values of $M, S$ and $A$.

\begin{tabular}{cccc}
\hline$M$ & $S$ & $A$ & $-f^{\prime \prime} 0$ \\
\hline 0.5 & 0.2 & 0.1 & 1.13992 \\
1.0 & 0.2 & 0.1 & 1.28430 \\
0.5 & 0.5 & 0.1 & 1.26830 \\
0.5 & 0.2 & 0.5 & 0.800331 \\
\hline
\end{tabular}

Table 3. Computations showing the local Nusselt number $-\theta(0)$ and Sherwood number $-\phi(0)$.

\begin{tabular}{cccc}
\hline$N b$ & $N t$ & $-\theta^{\prime} 0$ & $-\phi^{\prime} 0$ \\
\hline 0.1 & 0.1 & 0.0840676 & 2.96584 \\
0.5 & 0.1 & 0.0813944 & 3.00253 \\
0.1 & 0.5 & 0.0838497 & 2.79651 \\
0.5 & 0.5 & 0.0811044 & 2.97637 \\
\hline
\end{tabular}


Table 4. Computations showing the local Nusselt number $-\theta^{\prime} \quad 0$ and Sherwood number $-\phi^{\prime} 0$.

\begin{tabular}{cccccc}
\hline $\operatorname{Pr}$ & $R$ & $B i$ & $L e$ & $-\theta^{\prime} 0$ & $-\phi^{\prime} 0$ \\
\hline 1 & 0.5 & 0.1 & 5 & 0.0814229 & 2.97413 \\
2 & 0.5 & 0.1 & 5 & 0.0840344 & 2.95762 \\
1 & 1.0 & 0.1 & 5 & 0.0803668 & 2.98174 \\
1 & 0.5 & 1.0 & 5 & 0.28982 & 2.90082 \\
1 & 0.5 & 0.1 & 10 & 0.0811004 & 5.62347 \\
\hline
\end{tabular}

Fig. 2 reveals the influences of magnetic field on the flow field. The presence of transverse magnetic field sets in Lorentz force effect, which results in the retarding effect on the velocity field. As the values of magnetic parameter $M$ increase, the retarding force increases and consequently the velocity decreases. The graph also reveals that the boundary layer thickness reduces as magnetic parameter $M$ increases. The influence of the magnetic field parameter $M$ on the temperature and concentration are shown in Figs 3 and 4. On observing these figures, the temperature and concentration increases with the increase in magnetic field parameter. Furthermore, with an increase in $M$, the thermal boundary layer as well as concentration boundary layer decreases.

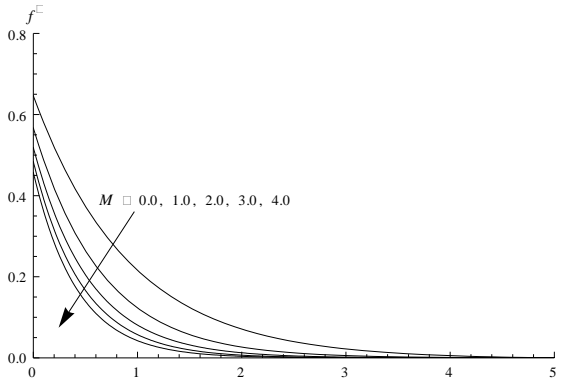

Fig. 2. Velocity profiles for different values of $M$.

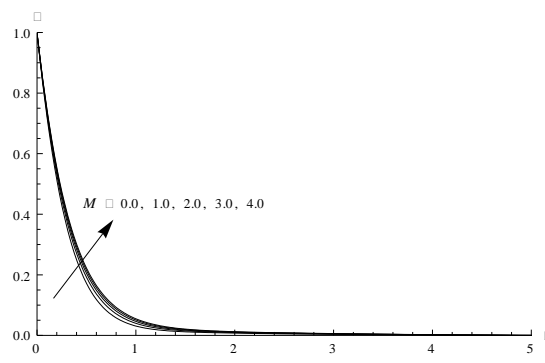

Fig. 4. Concentration profiles for different values of $M$.

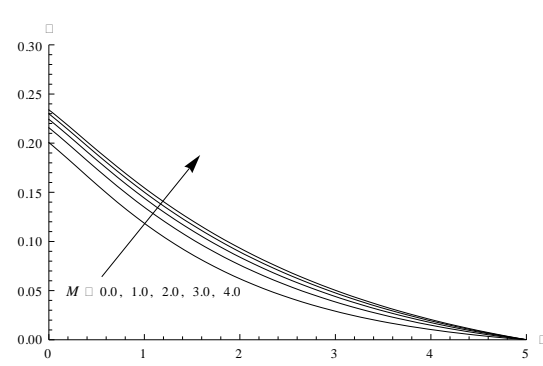

Fig. 3. Temperature profiles for different values of $M$.

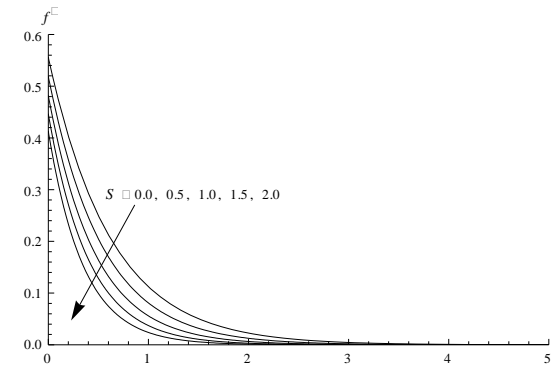

Fig. 5. Velocity profiles for different values of $S$. 
Fig. 5 shows the distinction of velocity profile with respect to the variation in suction parameter $S$. On observing these figure, as the values of $S$ increase, the velocity profile graph decreases. Fig. 6 displays the variation of temperature with suction parameter $s$. As the values of suction parameter $s$ increase, the temperature graph is decreasing. Moreover, the thermal boundary layer thickness and surface temperature is also decreasing. On the other hand the suction parameter $s$ has a strong influence on the concentration profile as it is shown in Fig. 7. As the values of suction parameter $s$ increase, concentration graph decreases and the concentration boundary layer thickness decreases.

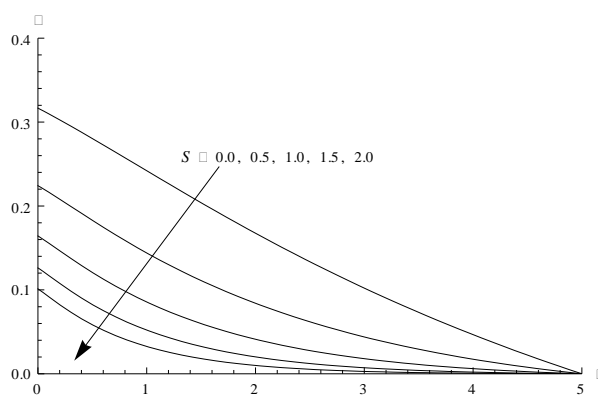

Fig. 6. Temperature profiles for different values of $S$.

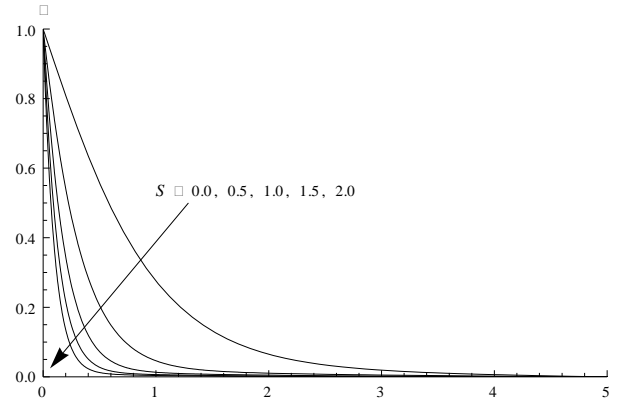

Fig. 7. Concentration profiles for different values of $S$.

For various values of the velocity slip parameter $_{A}$, the velocity profiles are plotted in Fig. 8. It is observed that the velocity graph decreases as the values of velocity slip parameter $A$ increase.

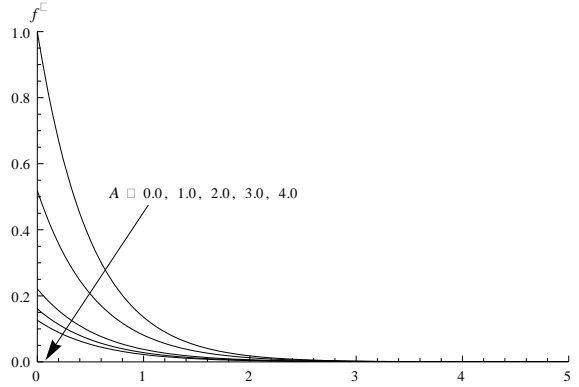

Fig. 8. Velocity profiles for different values of A.

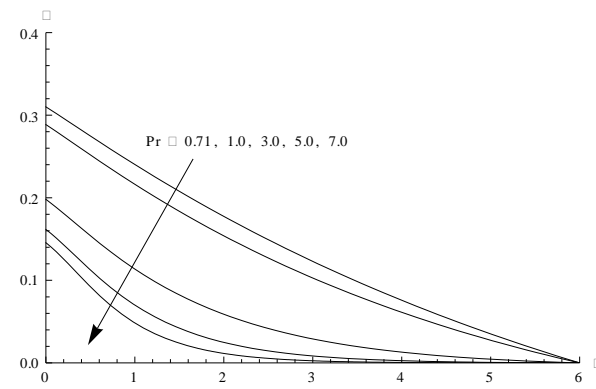

Fig. 9. Temperature profiles for different values of Pr.

Fig. 9 illustrates the temperature profiles for different values of the Prandtl number Pr . The Prandtl number defines the ratio of momentum diffusivity to thermal diffusivity. This figure reveals that an increase in Prandtl number $\operatorname{Pr}$ results in a decrease in the temperature distribution, because, thermal boundary layer thickness decreases with an 
increase in Prandtl number Pr . In short, an increase in the Prandtl number means slow rate of thermal diffusion. The graph also shows that as the values of Prandtl number Pr increase, the wall temperature decreases. The effect of Prandtl on a nanofluid is similar to what has already been observed in common fluids qualitatively but they are different quantitatively. Therefore, these properties are inherited by nanofluids.

The effect of radiation parameter on dimensionless temperature profiles is shown in Fig. 10. It is seen that as the radiation parameter increases, the thickness of the thermal boundary layer increases.

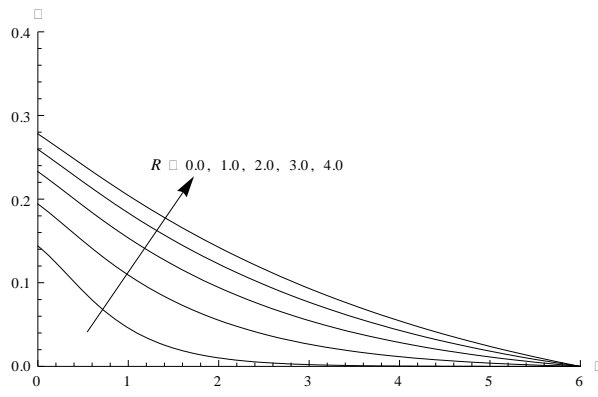

Fig. 10. Temperature profiles for different values of $R$.

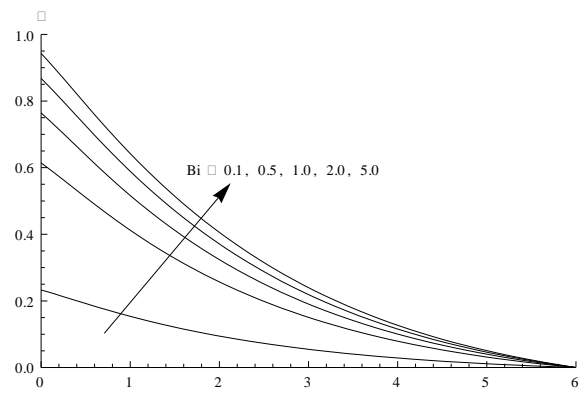

Fig. 11. Temperature profiles for different values of $B i$.

The influence of the Biot number $B i$ on the temperature and concentration profiles are plotted in Figures 11 and 12 respectively. It is observed that temperature field $\theta$ increases rapidly near the boundary by increasing Biot number. It was observed in Fig. 11 that as the convective heating of the sheet is enhanced i.e. Bi increases, the thermal penetration depth increases. Because the concentration distribution is driven by the temperature field, one anticipates that a higher Biot number would promote a deeper penetration of the concentration. This anticipation is indeed realized in Fig. 12 which predicts higher concentrations at higher values of the Biot number. Fig. 13 shows the temperature distributions in the thermal boundary layer for different values of the Brownian motion and the thermophoresis parameters. It is noticed that as thermophoesis parameter increases the thermal boundary layer thickness increases and the temperature gradient at the surface decrease (in absolute value) as both $N b$ and $N t$ increase. Fig. 14 reveals the variation of concentration in response to a change in Brownian motion parameter $\mathrm{Nb}$. As the values of Brownian motion parameter increase, the concentration boundary layer thickness is decreasing. Fig. 15 illustrates the variation of concentration graph in response to a change in thermophoresis parameter $N$. The influence of thermophoresis parameter on concentration profile graph is monotonic, i.e. as the values of $N_{t}$ parameter increase, the concentration boundary layer thickness is also increasing. 


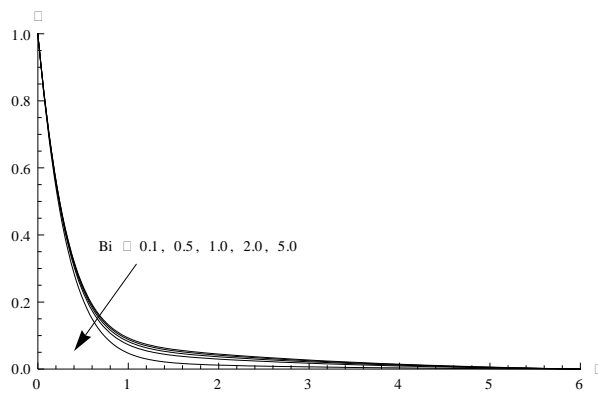

Fig. 12. Concentration profiles for different values of $\mathrm{Bi}$.

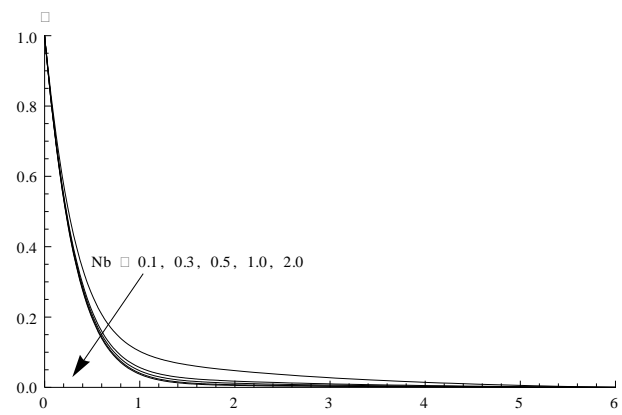

Fig. 14. Concentration profiles for different values of $\mathrm{Nb}$.

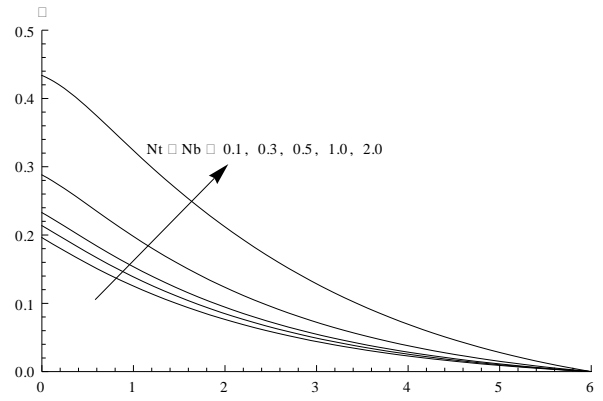

Fig. 13. Temperature profiles for different values of $\mathrm{Nb}$ and $\mathrm{Nt}$.

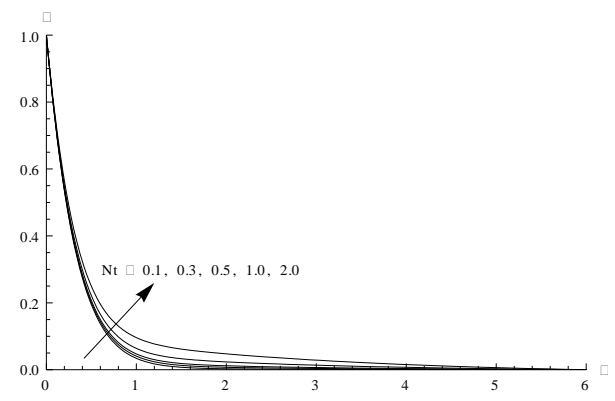

Fig. 15. Concentration profiles for different values of $N t$.

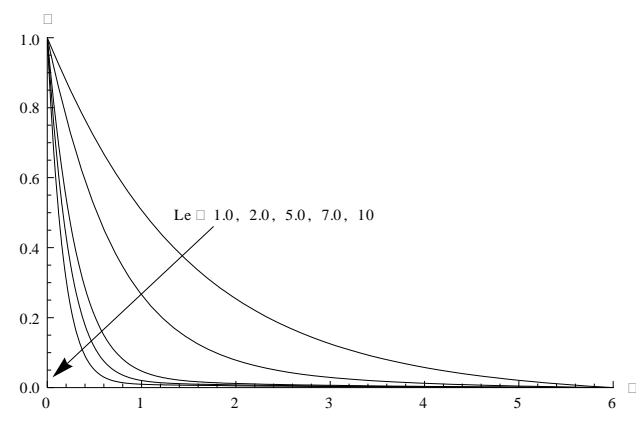

Fig. 16. Concentration profiles for different values of $L e$.

The influence of the Lewis number on the nanoparticle concentration profiles is presented in Fig. 16. It is clear that the Lewis number significantly affects the concentration distribution. As it is noticed from Fig. 16, as Lewis number increases the concentration graph decreases and the concentration boundary layer thickness decreases. 
This is probably due to the fact that mass transfer rate increases as Lewis number increases. It also reveals that the concentration gradient at surface of the sheet increases. Moreover, the concentration at the surface of a sheet decreases as the values of $L e$ increase.

Figs. 17-19 show the influence of governing parameters on dimensionless heat transfer rates, skin friction coefficient and dimensionless nanoparticle fraction rates. In Fig. 17, it depicts that for higher values of $M$, skin friction coefficient presents the increasing behavior corresponding to the increasing values of $s$. This shows that fluid motion on the wall of the sheet accelerated, when we strengthen the effects of parameter. From Fig. 18, it is noticed that local Nusselt number increases for increasing values of $\mathrm{R}$ and $\mathrm{Bi}$. Fig. 19 shows the variation in dimensionless nanoparticle fraction rates against $\mathrm{Pr}$ and Le, respectively. As Pr increase the mass rate increase.

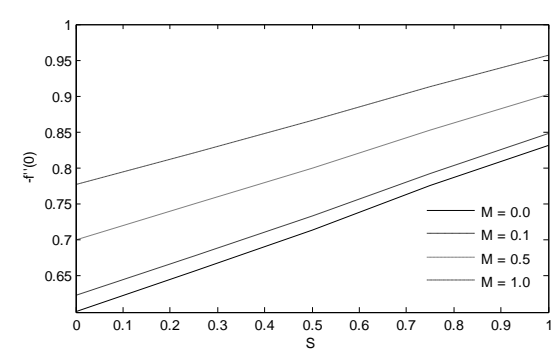

Fig. 17. Effects of $M$ and $S$ on Skin friction coefficient.

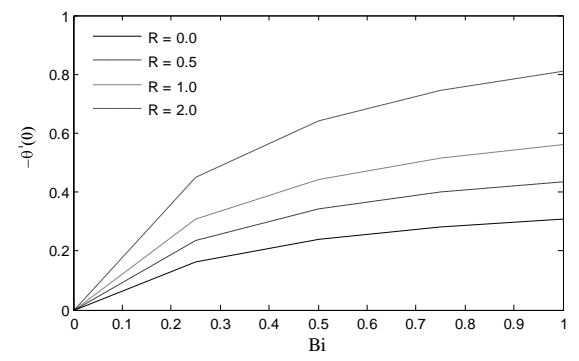

Fig. 18. Effects of $R$ and $B i$ on reduced Nusselt number.

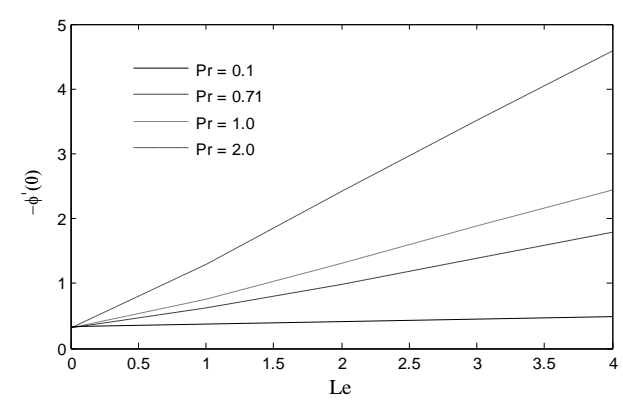

Fig. 19. Effects of Pr and Le on reduced Sherwood number.

\section{Conclusions}

A numerical study of the boundary layer flow in a nanofluid induced as a result of the motion of a linearly stretching sheet in the presence of heat radiation and MHD have been performed. The use of a velocity slip condition and convective heating boundary 
condition instead of a constant temperature or a constant heat flux makes this study more general novel. A similarity transformation is been used to transformed the governing partial differential equations into ordinary differential equations, a more convenient form for numerical computation. These equations are solved numerically using the shooting method alongside with fourth order of Runge-Kutta integration scheme. The main findings of the study are summarized as follows:

a. The thickness of velocity boundary layer decreases with an increase in magnetic field parameter $M$.

b. Velocity profiles decrease with an increase in $M$.

c. The velocity at the surface of a sheet decreases as the values of $A$ increase.

d. Thermal boundary layer thickness decreases with an increase in values of Prandtl number Pr .

e. The thickness of thermal boundary layer increases with an increase in radiation parameter $R$, magnetic field parameter $M$ and thermophoresis parameter ${ }_{N}$ when $N b=N t$.

f. With the increase in $B i$, the concentration layer thickens but the concentration layer becomes thinner as $L e$ increases.

\section{Acknowledgement}

The author wishes to express sincere thanks to referees for their valuable comments and suggestions for the improvement of the manuscript.

$\begin{array}{ll}\text { Nomenclature } \\ A & \text { velocity slip parameter } \\ a & \text { a positive constant associated with linear } \\ & \text { stretching } \\ B i & \text { Biot number } \\ C & \text { nanoparticle volume fraction } \\ C_{w} & \text { nanoparticle volume fraction at the sheet } \\ & \text { surface (wall) } \\ C_{\infty} & \text { nanoparticle volume fraction at large } \\ & \text { values of y (ambient) } \\ C_{f} & \text { skin friction coefficient } \\ D_{B} & \text { Brownian diffusion coefficient } \\ D_{T} & \text { thermophoretic diffusion coefficient } \\ f & \text { dimensionless stream function } \\ g & \text { acceleration due to gravity } \\ h & \text { convective heat transfer coefficient } \\ k & \text { thermal conductivity of base fluid } \\ k & \text { mean absorption coefficient. } \\ L e & \text { Lewis number } \\ M & \text { magnetic parameter }\end{array}$

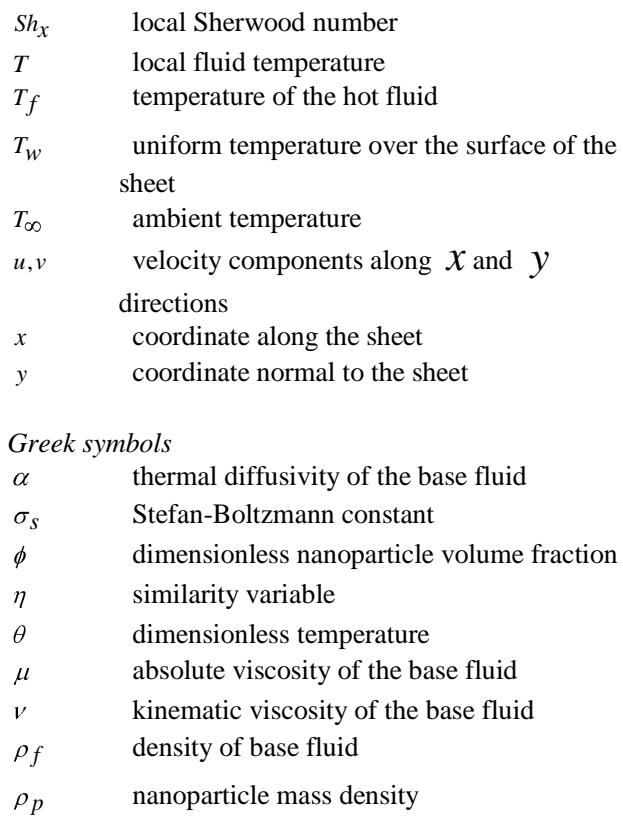




\begin{tabular}{llll}
$N b$ & Brownian motion parameter & $\rho c_{f}$ & heat capacity of the base fluid \\
$N t$ & thermophoresis parameter & $\rho c_{p} \quad$ heat capacity of the nanoparticle material \\
$N u_{x}$ & local Nusselt number & $\tau$ & $\rho c_{p} / \rho c_{f}$ \\
$\operatorname{Pr}$ & Prandtl number & $\psi$ & stream function \\
$p$ & pressure & Subscripts \\
$q_{r}$ & radiative heat flux & $\infty$ & condition at the free stream \\
$q_{m}^{\prime \prime}$ & wall mass flux & $w$ & condition at the surface \\
$q_{w}^{\prime \prime}$ & wall heat flux & \multicolumn{2}{l}{} \\
$\operatorname{Re}_{x}$ & local Reynolds number &
\end{tabular}

\section{References}

1. H. S. Takhar, A.J. Chamkha, and G. Nath, Acta Mech. 146, 59 (2001). http://dx.doi.org/10.1007/BF01178795

2. J. Vleggaar, Chem. Eng. Sci. 32, 1517 (1977). http://dx.doi.org/10.1016/0009-2509(77)80249-2

3. L. J. Crane, J. Appl. Math. Phys (ZAMP) 21, 645 (1970). http://dx.doi.org/10.1007/BF01587695

4. K. N. Lakshmisha, S. Venkateswaran, and G. Nath, ASME J. Heat Transfer 110, 590 (1988). http://dx.doi.org/10.1115/1.3250533

5. C. Y. Wang, Phys. Fluids 27, 1915 (1984). http://dx.doi.org/10.1063/1.864868

6. H. I. Andersson and B. S. Dandapat, SAACM 1, 339 (1991).

7. E. Magyari and B. Keller, Eur. J. Mech. B Fluids 19, 109 (2000). http://dx.doi.org/10.1016/S0997-7546(00)00104-7

8. E. M. Sparrow and J. P. Abraham, Int. J. Heat Mass Transfer 48, 3047 (2005). http://dx.doi.org/10.1016/j.ijheatmasstransfer.2005.02.028

9. J. P. Abraham and E. M. Sparrow, Int. J. Heat Fluid Flow 26, 289 (2005). http://dx.doi.org/10.1016/j.ijheatfluidflow.2004.08.007

10. P. Keblinski, J. A. Eastman, and D. G. Cahaill, Nanofluids for thermal transport, Mater Today 8 (6), 36 (2005). http://dx.doi.org/10.1016/S1369-7021(05)70936-6

11. S. Choi, Enhancing Thermal Conductivity of Fluids with Nanoparticles, Developments and Applications of Non-Newtonian Flows, D.A. Siginer and H.P. Wang (eds,) FED-Vol. 231/MDVol. 66, ASME, New York, 99 (1995) pp 99-105.

12. J. A. Eastman, S. U. S. Choi, S. Li S, W. Yu, and L. J. Thompson, Appl. Phys. Lett. 78 (6), 718 (2001). http://dx.doi.org/10.1063/1.1341218

13. S. U. S. Choi, Z. G. Zhang, W. Yu, and F. E. Lockwoow, Appl. Phys. Lett. 79, 2252 (2001). http://dx.doi.org/10.1063/1.1408272

14. A. V. kuznetsov and D. A Int. J. Therm. Sci. 49, 243 (2010).

15. W. A. Khan and I. Pop, Int. J. Heat Mass Transfer 53, 2477 (2010). http://dx.doi.org/10.1016/j.ijheatmasstransfer.2010.01.032

16. W. Ibrahim and B. Shanker, J Fluid Eng-Trans ASME 134, 081203-1 (2012). http://dx.doi.org/10.1115/1.4007075

17. Z. Haddad, A. Nada, F. Oztop, and A. Mataoui, Int. J. Therm. Sci. 57, 152 (2012). http://dx.doi.org/10.1016/j.ijthermalsci.2012.01.016

18. O. D. Makinde and A. Aziz, Int. J. Therm. Sci. 50, 1326 (2011). http://dx.doi.org/10.1016/j.ijthermalsci.2011.02.019

19. M. G. Reddy, ISRN thermodynamics 2012, 1 (2012).

20. P. O. Olanrewaju, M. A. Olanrewaju, and A. O. Adesanya, Int. J. Appl. Sci. Tech. 2 (1), 274 (2012).

21. M. G. Reddy, Acta Technica 1, 41 (2013).

22. C. Y. Wang, J. Appl. Math. Mech. (ZAMM) 69, 418 (1989).

23. N. S. Akbar, S. Nadeem, R. Ul Haq Z. H. Khan, Chinese J. Aeronaut. 26 (6), 1389 (2013).

24. S. Nadeem, R. Ul Haq, Z. H. Khan, Alexandria Eng. J. (2013) (In press). 